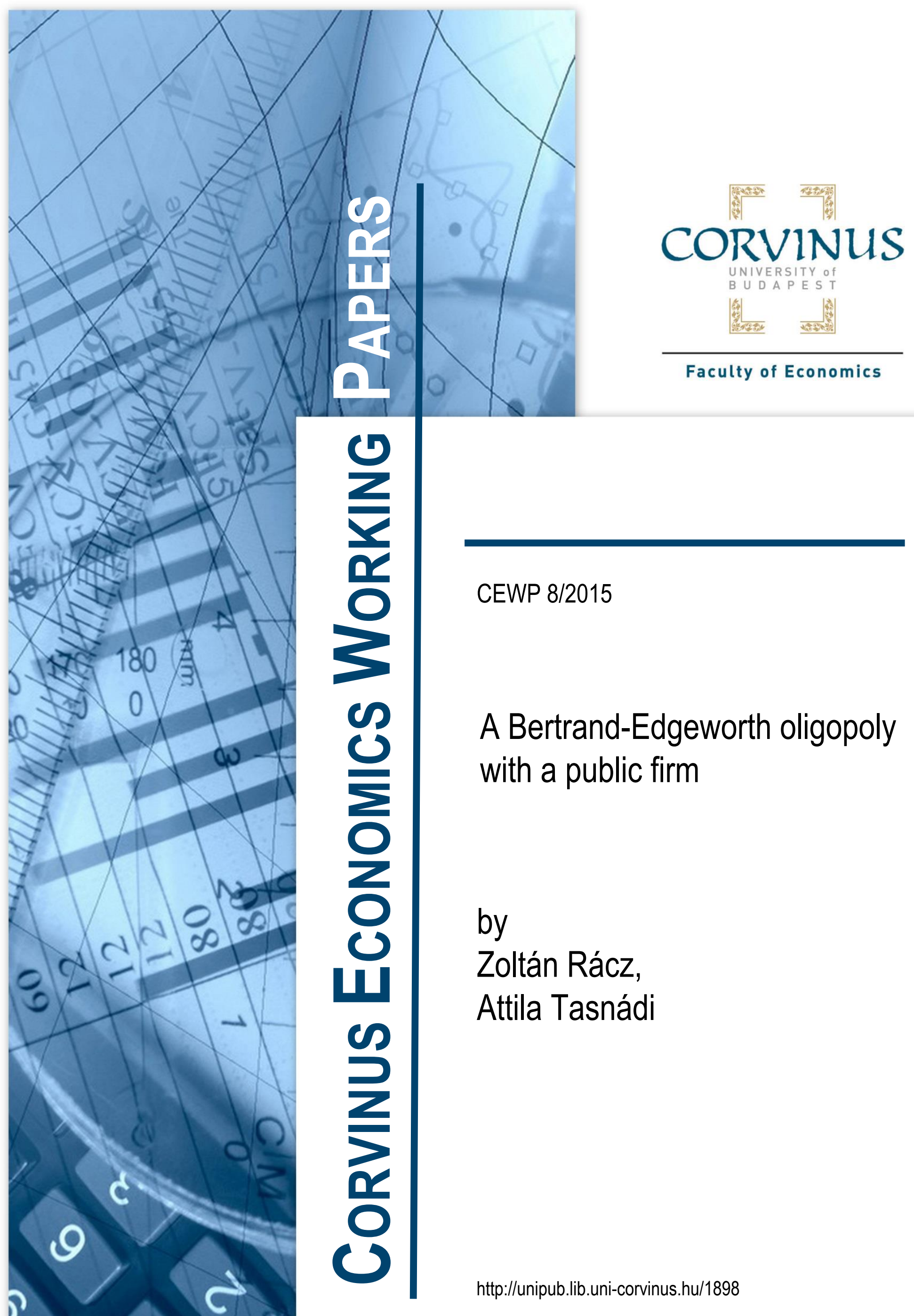




\title{
A Bertrand-Edgeworth oligopoly with a public firm
}

\author{
Zoltán Rácz \\ Corvinus University of Budapest* \\ Attila Tasnádi \\ Corvinus University of Budapest ${ }^{\dagger}$
}

April 7, 2015

\begin{abstract}
We determine conditions under which a pure-strategy equilibrium of a mixed Bertrand-Edgeworth oligopoly exists. In addition, we determine its pure-strategy equilibrium whenever it exists and compare the equilibrium outcome with that of the standard Bertrand-Edgeworth oligopoly with only private firms.
\end{abstract}

Keywords: Bertrand-Edgeworth, mixed oligopoly.

JEL Classification Number: D43, L13.

\section{Introduction}

In mixed oligopolies a public firm, which maximizes social surplus, competes with at least one private firm. The literature on mixed oligopolies focused on quantity-setting games (e.g. De Fraja and Delbono (1989), and Matsumura and Okumura (2013)) or on heterogeneous goods price-setting games (e.g. Bárcena-Ruiz (2007)). A homogeneous good price-setting mixed duopoly was investigated by Balogh and Tasnádi (2012).

Probably the mixed homogeneous good price-setting oligopoly has not been analyzed so far because of the difficulties in characterizing and determining the equilibrium of its standard counterpart with purely private firms. Although the equilibrium of the relatively simple symmetric oligopoly game with identical firms was solved by Vives (1986), the equilibrium of the general triopolistic case was obtained only recently, independently by Hirata (2009) and De Francesco and Salvadori (2010). In case of $n \geq 4$, the full characterization of the equilibrium is still undone but some of its important properties were derived by De Francesco and Salvadori (2010), which enable us to compare the purely private oligopoly with the mixed oligopoly in terms of social surplus.

While in the homogeneous good price-setting game with purely private firms a purestrategy equilibrium fails to exist for a wide range of capacities (see, for instance, Dasgupta

\footnotetext{
*e-mail: zoltan.racz@stud.uni-corvinus.hu

† (corresponding author) MTA-BCE "Lendület" Strategic Interactions Research Group, Department of Mathematics, H-1093 Budapest, Fővám tér 8. e-mail: attila.tasnadi@uni-corvinus.hu
} 
and Maskin (1986) and Vives (1986)) we show that its mixed version has a pure-strategy equilibrium for a much wider range of capacities. In addition, if the price-setting oligopoly game with purely private firms has an equilibrium in non-degenerated mixed strategies, we prove that the presence of a public firm is strictly social surplus increasing whenever in the latter case a pure-strategy equilibrium exists, which holds if the public firm is sufficiently large. Besides extending the results obtained by Balogh and Tasnádi (2012) from duopolies to oligopolies another interesting feature of having more than two firms in the market is that one Pareto inferior type of Nash equilibrium vanishes when we move from two to more firms.

The remainder of the paper is organized as follows. Section 2 introduces the framework, in Section 3 we recall the results of De Francesco and Salvadori (2010), which will serve as a benchmark in our analysis. Section 4 contains the characterization of the pure-strategy equilibria.

\section{The framework}

The demand is given by function $D$ on which we impose the following restrictions:

Assumption 1. $D$ intersects the horizontal axis at quantity $a$ and the vertical axis at price $b . D$ is strictly decreasing, concave and twice continuously differentiable on $(0, b)$; moreover, $D$ is right-continuous at 0 , left-continuous at $b$ and $D(p)=0$ for all $p \geq b$.

On the producers' side we have a public firm and $n \geq 2$ private firms. We label the public firm with 0 and the private firms with $1,2, \ldots, n$. Our assumptions imposed on the firms' cost functions are as follows:

Assumption 2. The firms have zero unit costs up to their respective positive capacity constraints $k_{0}, k_{1}, \ldots, k_{n}$, where $k_{1} \leq k_{2} \leq \ldots \leq k_{n}{ }^{1}$

We shall denote by $P$ the inverse demand function and by $p^{c}$ the market clearing price, i.e. $p^{c}=P\left(\sum_{i=0}^{n} k_{i}\right)$ and $p_{0}, p_{1}, \ldots, p_{n} \in[0, b]$ stand for the prices set by the firms.

For all $i \in\{0,1, \ldots, n\}$ we shall denote by $p_{i}^{m}$ the unique revenue maximizing price on the firm's residual demand curve $D_{i}^{r}(p)=\left(D(p)-\sum_{j=0, j \neq i}^{n} k_{j}\right)^{+}$taking the capacity constraint into account, i.e. $p_{i}^{m}=\arg \max _{p \in[0, b]} p \cdot \min \left\{D_{i}^{r}(p), k_{i}\right\}$.

Let us denote by $p_{i}^{d}$ the smallest price for which $p_{i}^{d} \cdot \min \left\{k_{i}, D\left(p_{i}^{d}\right)\right\}=p_{i}^{m} D_{i}^{r}\left(p_{i}^{m}\right)$ for each $i \in\{0,1, \ldots, n\}$. Provided that private firm $i$ has 'sufficient' capacity (i.e. $p^{c}<p_{i}^{m}$ ), then if it is a profit-maximizer, it is indifferent to whether serving residual demand at price level $p_{i}^{m}$ or selling $\min \left\{k_{i}, D_{i}\left(p_{i}^{d}\right)\right\}$ at the lower price level $p_{i}^{d}$. Clearly, $p^{c}, p_{i}^{m}$ and $p_{i}^{d}$ are well-defined, and it can be verified that $p^{c} \leq p_{i}^{d} \leq p_{i}^{m}, p_{i}^{m} \geq p_{j}^{m}$ and $p_{i}^{d} \geq p_{j}^{d}$ hold, whenever Assumptions 1 and 2 are satisfied and $1 \leq i \leq j \leq n$.

Now we define $\widehat{p}^{c}, \widehat{p}_{i}^{m}$ and $\widehat{p}_{i}^{d}$ in the same way as $p^{c}, p_{i}^{m}$ and $p_{i}^{d}$, except that we assume that the public firm does not enter the market, i.e. $\widehat{p}^{c}=P\left(\sum_{i=1}^{n} k_{i}\right), \widehat{p}_{i}^{m}=$ $\arg \max _{p \in[0, b]} p \cdot \min \left\{\widehat{D}_{i}^{r}(p), k_{i}\right\}$, where $\widehat{D}_{i}^{r}(p)=\left(D(p)-\sum_{j=1, j \neq i}^{n} k_{j}\right)^{+}$and $\widehat{p}_{i}^{d}$ is the smallest price for which $\widehat{p}_{i}^{d} \cdot \min \left\{k_{i}, D\left(\widehat{p}_{i}^{d}\right)\right\}=\widehat{p}_{i}^{m} \widehat{D}_{i}^{r}\left(\widehat{p}_{i}^{m}\right)$. Note that $p^{c} \leq \widehat{p}^{c}$ and $p_{i}^{d} \leq \widehat{p}_{i}^{d}$ for any $i \in\{1,2, \ldots, n\}$.

We assume that the firms play the production-to-order type Bertrand-Edgeworth game, and therefore, the game reduces to a price-setting game since the firms can adjust their

\footnotetext{
${ }^{1}$ The real assumption here is that firms have identical unit costs since in case of production-to-order, as will be assumed later, this is just a matter of normalization.
} 
productions to the demands they face. Regarding the employed rationing rule and tiebreaking rule, we impose the following assumptions.

Assumption 3. We assume efficient rationing on the market.

Assumption 4. We assume that in case of price ties the firms setting identical prices divide the residual demand in proportion to their capacities. However, each firm has the option of giving up a part or the whole of its portion of residual demand in favor of the other firms setting the same price.

It is clear that no profit-maximizing firm will give up the demand it is entitled to up to its capacity constraint. Nevertheless, under certain circumstances, as we will see later, the public firm can lead the market to a socially better equilibrium by restricting its supply at 'sufficiently' low price levels since it may discourage private firms matching its price from raising their prices. For each firm $i \in\{1,2, \ldots, n\}$, let $E_{i}$ mean the set of those firms which set the same price as firm $i$. The definition of $L_{i}$ for firms setting lower prices is analogous, so $E_{i}=\left\{j \mid p_{j}=p_{i}, j=0,1, \ldots, n\right\}$ and $L_{i}=\left\{j \mid p_{j}<p_{i}, j=0,1, \ldots, n\right\}$.

Thus, if $\mathbf{p}=\left(p_{0}, p_{1}, \ldots, p_{n}\right), \mathbf{k}=\left(k_{0}, k_{1}, \ldots, k_{n}\right)$ and $\Delta_{0}(D, \mathbf{p}, \mathbf{k})$ stands for the public firm's served demand, then

$$
\min \left\{k_{0},\left(D\left(p_{0}\right)-\sum_{l \in L_{0}} k_{l}-\sum_{\substack{j \in E_{0} \\ j \neq 0}} k_{j}\right)^{+}\right\} \leq \Delta_{0}(D, \mathbf{p}, \mathbf{k}) \leq \min \left\{k_{0}, \frac{k_{0}}{\sum_{j \in E_{0}} k_{j}}\left(D\left(p_{0}\right)-\sum_{l \in L_{0}} k_{l}\right)^{+}\right\}
$$

must hold. Essentially, $\Delta_{0}(D, \mathbf{p}, \mathbf{k})$ describes the public firm's behavior of letting or not letting other firms serve its market share. According to Assumptions 3 and 4, the demand served by private firm $i \in\{1,2, \ldots, n\}$ is given by

$$
\Delta_{i}(D, \mathbf{p}, \mathbf{k})= \begin{cases}\min \left\{k_{i}, \frac{k_{i}}{\sum_{j \in E_{i}} k_{j}}\left(D\left(p_{i}\right)-\sum_{l \in L_{i}} k_{l}\right)^{+}\right\} & \text {if } 0 \notin E_{i}, \\ \min \left\{k_{i}, \frac{k_{i}}{\sum_{j \in E_{i}} k_{j}-k_{0}}\left(D\left(p_{i}\right)-\sum_{l \in L_{i}} k_{l}-\Delta_{0}(D, \mathbf{p}, \mathbf{k})\right)^{+}\right\} & \text {if } 0 \in E_{i} .\end{cases}
$$

We shall denote by $S_{\mathbf{p}}(p)=\sum_{\left\{i \mid p_{i} \leq p\right\}} k_{i}$ the supply curve and by $p_{\mathbf{p}}^{s}=$ $\min \left\{p \in[0, b] \mid D(p) \leq S_{\mathbf{p}}(p)\right\}$ the price determining social surplus for any given price profile $\mathbf{p}$. The public firm aims to maximize total surplus given by

$$
\pi_{0}(\mathbf{p})=\int_{0}^{D\left(p_{\mathbf{p}}^{s}\right)} P(q) d q
$$

As the private firms are profit-maximizers, their object function is

$$
\pi_{i}(\mathbf{p})=p_{i} \Delta_{i}(D, \mathbf{p}, \mathbf{k}) .
$$

\section{The benchmark}

The following results concerning the purely private price-setting oligopoly will serve as a benchmark:

Proposition 1 (De Francesco and Salvadori (2010)). For the purely private price-setting oligopoly under Assumptions 1-4, the following statements are known. 
1. The only pure-strategy equilibrium of the game can be $\left\{p^{c}, p^{c}, \ldots, p^{c}\right\}$, which is an equilibrium if and only if $\max \left\{p_{0}^{d}, p_{1}^{d}\right\}=p^{c}$.

2. Even in case of a mixed-strategy equilibrium, each firm will set a price equal to or larger than $\max \left\{p_{0}^{d}, p_{1}^{d}\right\}$. More precisely, $\max \left\{p_{0}^{d}, p_{1}^{d}\right\}$ is the lowest of the supports' minima of the firms' strategies.

Note that the second point necessarily means that in any mixed-strategy equilibrium the social surplus must be lower than in a strategy profile where each firm sets price $p_{1}^{d}$.

\section{Characterization of the pure-strategy equilibria}

We analyze the following two-stage game: in the first stage the public firm choses a suitable $\Delta_{0}(D, \mathbf{p}, \mathbf{k})$ (a strategy to decide when to share its demand with the private firms in the second stage); in the second stage, after the private firms have been informed about $\Delta_{0}(D, \mathbf{p}, \mathbf{k})$, a price competition takes place.

Lemma 1. Under Assumptions 1-4 and $p_{1}^{d}>0$, price $p_{\mathbf{p}}^{s}$ must be positive in any purestrategy equilibrium if such an equilibrium exists.

Proof. Assume that $p_{1}^{d}>0$ and there is a pure-strategy equilibrium in which $p_{\mathbf{p}}^{s}=0$. Since $p_{1}^{d}>0$ implies $\sum_{j=0, j \neq 1}^{n} k_{j}<a$, and therefore it follows that firm 1 could still sell a positive amount at a positive price; a contradiction.

From now on, we restrict ourselves to the case when $p_{1}^{d}>p^{c}$, since when $p_{1}^{d}=$ $p^{c}$ any pure-strategy equilibrium is payoff-equivalent to the market-clearing outcome $\left(p^{c}, p^{c}, \ldots, p^{c}\right)$, similarly to the purely private case.

Assumption 5. We only consider $\mathbf{k}$ and $D(p)$ for which $p_{1}^{d}>p^{c}$.

Lemma 2. Under Assumptions 1-5 all private firms must set the same price in any purestrategy equilibrium.

Proof. Assume that the private firms set at least two different prices in a pure-strategy equilibrium p. Consider one of the private firms with the highest price: If its residual demand is zero, its profit is also zero, and it could benefit by setting a positive price below $p_{\mathbf{p}}^{s}$. If its residual demand is positive, any of the firms setting a lower price could increase its profits by setting its price anywhere between the current highest and second highest prices, hence $\mathbf{p}$ cannot be an equilibrium.

Henceforth, we shall denote by $p^{*}$ the common pure-strategy equilibrium price of the private firms if such an equilibrium exists.

Lemma 3. Under Assumptions 1-5 in any pure-strategy equilibrium $\left(p_{0}, p^{*}, p^{*}, \ldots, p^{*}\right)$ we must have that all the private firms sell their entire capacities.

Proof. Otherwise the profit could be increased by slightly undercutting $p^{*}$.

Lemma 4. Under Assumptions 1-5 in any pure-strategy equilibrium $\left(p_{0}, p^{*}, p^{*}, \ldots, p^{*}\right)$ we must have that $D\left(p^{*}\right) \leq \sum_{p_{j} \leq p^{*}} k_{j}$.

Proof. Otherwise each private firm could increase its profit by slightly rising its price. 
We are now ready to specify the pure-strategy equilibria with respect to an arbitrary $\Delta_{0}(D, \mathbf{p}, \mathbf{k})$.

Proposition 2. Assume that Assumptions 1-5 hold. Then the simultaneous-move game can only have the following two types of pure-strategy equilibria:

$$
p_{0}=p^{*}=P\left(\sum_{j=1}^{n} k_{j}+\Delta_{0}\left(D, \mathbf{p}^{*}, \mathbf{k}\right)\right) \quad\left(P S E_{1}\right),
$$

where $\mathbf{p}^{*}=\left\{p^{*}, p^{*}, \ldots, p^{*}\right\}$ and which is an equilibrium if and only if $p_{1}^{d} \leq p^{*} \leq P\left(k_{0}\right)$, $\Delta_{0}\left(D, \mathbf{p}^{*}, \mathbf{k}\right)=D\left(p^{*}\right)-\sum_{j=1}^{n} k_{j}$ and $p_{1}^{d} \leq \hat{p}^{c} .^{2}$ In addition, the strategy profile

$$
p^{*}=\widehat{p}^{c} \quad \text { and } p_{0}>p^{*}\left(P S E_{2}\right),
$$

is an equilibrium if and only if $\widehat{p}_{1}^{d}=\widehat{p}^{c} \leq P\left(k_{0}\right)$.

Proof. Assume firstly that $p_{0}<p^{*}$. In this case $p^{*}=p^{c}$ is the only possible equilibrium price due to Lemmas 3 and 4 . However, since $p^{c}<p_{1}^{d}$ because of Assumption 5, playing $p_{1}^{m}$ instead of $p^{c}$ would be strictly better for firm 1 , hence no such pure-strategy equilibrium can exist.

Now consider the case $p_{0}=p^{*}$. Clearly only $p^{*}=P\left(\sum_{j=1}^{n} k_{j}+\Delta_{0}\left(D, \mathbf{p}^{*}, \mathbf{k}\right)\right)$ with the condition $\Delta_{0}\left(D, \mathbf{p}^{*}, \mathbf{k}\right)=D\left(p^{*}\right)-\sum_{j=1}^{n} k_{j} \geq 0$ satisfies Lemmas 3 and 4 . The public firm cannot increase social surplus, unless it is able to lower the private firms' residual demand to 0 by undercutting $p^{*}$, which is possible if and only if $P\left(k_{0}\right)<p^{*}$. As the private firms sell their entire capacities, they would not profit by lowering their prices. It is easy to see, that due to the concavity of the demand function, any firm $i \in\{1,2, \ldots, n\}$ can increase its profit by rising its price if and only if $p_{i}^{d}>p^{*}$.

When $p_{0}>p^{*}, p^{*}=\widehat{p}^{c}$ is the only price that satisfies Lemmas 3 and 4 . Condition $p^{*} \leq P\left(k_{0}\right)$ can be justified as in the previous case. The condition preventing the private firms from switching to a higher price is also similar, but here we have to use $\widehat{p}_{i}^{d}$ instead of $p_{i}^{d}$ as the public firm does not compete with private firms unless they set a higher price than $p_{0}$.

Observe that since $p_{i}^{d} \leq \widehat{p}_{i}^{d}, P S E_{1}$ always exists with an appropriately chosen $\Delta_{0}$ at price $p^{*}$ when $P S E_{2}$ does. We consider $P S E_{2}$ as an unlikely equilibrium, since it implies that the public firm does not enter the market despite knowing that another pure-strategy equilibrium with higher social surplus would be possible. Furthermore, any $P S E_{1}$ equilibrium price below $\widehat{p}^{c}$, if a pure-strategy equilibrium exists, weakly dominates any $P S E_{2}$ equilibrium price.

In order to maximize social surplus the public firm should choose $\Delta_{0}$ in a way that the $P S E_{1}$ type equilibrium price becomes as small as possible. Taking into consideration that $P\left(\sum_{j=1}^{n} k_{j}+\Delta_{0}(D, \mathbf{p}, \mathbf{k})\right) \in\left[p^{c}, \widehat{p}^{c}\right]$ is a function of $\Delta_{0}$, an optimal $\Delta_{0}$ for the public firm, resulting in equilibrium price $p_{1}^{d}$, is given by

$$
\Delta_{0}^{*}(D, \mathbf{p}, \mathbf{k})= \begin{cases}\min \left\{k_{0},\left(D\left(p_{0}\right)-\sum_{l \in L_{0}} k_{l}-\sum_{\substack{j \in E_{0} \\ j \neq 0}} k_{j}\right)^{+}\right\} & \text {if } \forall i: p_{i} \leq p_{1}^{d}, \\ \min \left\{k_{0}, \frac{k_{0}}{\sum_{j \in E_{0}} k_{j}}\left(D\left(p_{0}\right)-\sum_{l \in L_{0}} k_{l}\right)^{+}\right\} & \text {if } \exists i: p_{i}>p_{1}^{d},\end{cases}
$$

\footnotetext{
${ }^{2}$ The latter condition is equivalent with $D\left(p^{*}\right)-\sum_{j=1}^{n} k_{j} \geq 0$.
} 
where $i \in\{1,2, \ldots, n\}$.

$P S E_{1}$ simplifies to $p^{*}=p_{1}^{d}$ with only one necessary and sufficient condition in case of $\Delta_{0}^{*}$ since we can omit condition $P\left(\sum_{j=1}^{n} k_{j}+\Delta_{0}(D, \mathbf{p}, \mathbf{k})\right) \leq P\left(k_{0}\right)$ by $p_{i}^{d} \leq p_{i}^{m} \leq$ $P\left(\sum_{j \neq i} k_{j}\right) \leq P\left(k_{0}\right)$, which results in the following proposition.

Proposition 3. Assume that Assumptions 1-5 are satisfied and that the public firm choses $\Delta_{0}^{*}$. Then the simultaneous-move game can only have the following two types of purestrategy equilibria:

$$
p_{0}=p^{*}=p_{1}^{d}\left(P S E_{1}\right),
$$

which is an equilibrium if and only if $p_{1}^{d} \leq \widehat{p}^{c}$. In addition, the strategy profile

$$
p^{*}=\widehat{p}^{c} \quad \text { and } p_{0}>p^{*}\left(P S E_{2}\right),
$$

is an equilibrium if and only if $\widehat{p}_{1}^{d}=\widehat{p}^{c} \leq P\left(k_{0}\right)$.

Since under Assumptions 1-5 by Proposition 1 the purely private oligopoly has only a mixed-strategy equilibrium in which no firm sets a price below $\max \left\{p_{1}^{d}, p_{0}^{d}\right\}$, it follows for the respective mixed oligopoly that it has a strictly higher social surplus whenever it has a pure-strategy equilibrium and the $P S E_{1}$-type equilibrium will be played. We have already emphasized after Proposition 2 why a $P S E_{1}$-type equilibrium is more plausible than a $P S E_{2}$-type one.

Comparing our result with the duopolistic case investigated by Balogh and Tasnádi (2012), $P S E_{1}$ and $P S E_{2}$ correspond to their $N E_{1}$ and $N E_{3}$, respectively. Surprisingly, the Pareto inferior equilibrium $N E_{2}$ of the duopoly game vanishes in case of more than two private firms.

Finally, it can be easily checked that $k_{0} \geq k_{1}$ implies the existence of a pure-strategy equilibrium under Assumptions 1-5 and $\Delta_{0}^{*}$. Therefore, if the market is not competitive enough to have a pure-strategy equilibrium, the state can increase social welfare by acquiring a sufficiently large private firm and operating it as a public firm.

\section{Acknowledgements}

The second author gratefully acknowledges the financial support from the Hungarian Scientific Research Fund (K-101224).

\section{References}

BAlogh, T., And A. TASnádi (2012): "Price Leadership in a Duopoly with Capacity Constraints and Product Differentiation," Journal of Economics, 106, 233-249.

Bárcena-Ruiz, J. C. (2007): "Endogenous Timing in a Mixed Duopoly: Price Competition," Journal of Economics, 91, 263-272.

Dasgupta, P., and E. Maskin (1986): "The Existence of Equilibria in Discontinous Games II: Applications," Review of Economic Studies, 53, 27-41.

De Fraja, G., and F. Delbono (1989): "Alternative Strategies of a Public Enterprise in Oligopoly," Oxford Economic Papers, 41, 302-311. 
De Francesco, M., and N. Salvadori (2010): "Bertrand-Edgeworth Games under Oligopoly with a Complete Characterization for the Triopoly," MPRA Paper No. $2408 \%$.

Hirata, D. (2009): "Asymmetric Bertrand-Edgeworth Oligopoly and Mergers," B.E. Journal of Theoretical Economics, 9, "Article 22".

Matsumura, T., and Y. Okumura (2013): "Privatization neutrality theorem revisited," Economics Letters, 118, 324-326.

Vives, X. (1986): "Rationing Rules and Bertrand-Edgeworth Equilibria in Large Markets," Economics Letters, 21, 113-116. 